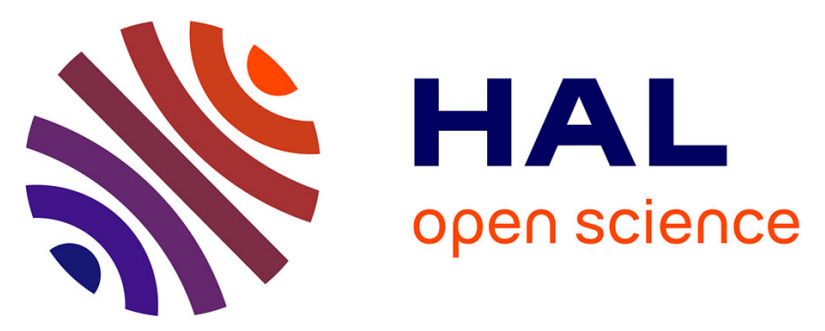

\title{
Energy-aware Georouting with Guaranteed Delivery in Wireless Sensor Networks with Obstacles
}

\author{
Essia Hamouda, Nathalie Mitton, Bogdan Pavkovic, David Simplot-Ryl
}

\section{To cite this version:}

Essia Hamouda, Nathalie Mitton, Bogdan Pavkovic, David Simplot-Ryl. Energy-aware Georouting with Guaranteed Delivery in Wireless Sensor Networks with Obstacles. International Journal of Wireless Information Networks, 2009, 16 (3), 10.1007/s10776-009-0105-1 . inria-00599171

\section{HAL Id: inria-00599171 \\ https://hal.inria.fr/inria-00599171}

Submitted on 8 Jun 2011

HAL is a multi-disciplinary open access archive for the deposit and dissemination of scientific research documents, whether they are published or not. The documents may come from teaching and research institutions in France or abroad, or from public or private research centers.
L'archive ouverte pluridisciplinaire HAL, est destinée au dépôt et à la diffusion de documents scientifiques de niveau recherche, publiés ou non, émanant des établissements d'enseignement et de recherche français ou étrangers, des laboratoires publics ou privés. 


\title{
Energy-aware Georouting with Guaranteed Delivery in Wireless Sensor Networks with Obstacles
}

\author{
E. H. Elhafsi ${ }^{1}$, N. Mitton ${ }^{2}$, B.Pavkovic ${ }^{2,3}$ and D. Simplot-Ryl ${ }^{2}$ \\ ${ }^{1}$ University of California, Riverside, CA, USA \\ ${ }^{2}$ INRIA Lille-Nord Europe, LIFL, Univ. Lille 1, France \\ ${ }^{3}$ Faculty of Technical Sciences, University of Novi Sad, Serbia \\ ${ }^{1}$ essia@cs.ucr.edu, ${ }^{2}\left\{\right.$ mitton,simplot-Ryl\}@lifl.fr, ${ }^{3}$ bogprs@gmail.com
}

July 3, 2009

\begin{abstract}
We propose, EtE, a novel end-to-end localized routing protocol for wireless sensor networks that is energy-efficient and guarantees delivery. To forward a packet, a node $s$ in graph $G$ computes the cost of the energy weighted shortest path between $s$ and each of its neighbors in the forward direction towards the destination which minimizes the ratio of the cost of the shortest path to the progress (reduction in distance towards the destination). It then sends the message to the first node on the shortest path from $s$ to $x$ : say node $x^{\prime}$. Node $x^{\prime}$ restarts the same greedy routing process until the destination is reached or an obstacle is encountered and the routing fails. To recover from the latter scenario, local minima trap, our algorithm invokes an energy-aware Face routing that guarantees delivery. Our work is the first to optimize energy consumption of Face routing. It works as follows. First, it builds a connected dominating set from graph $G$, second it computes its Gabriel graph to obtain the planar graph $G^{\prime}$. Face routing is invoked and applied to $G^{\prime}$ only to determine which edges to follow in the recovery process. On each edge, greedy routing is applied. This two-phase (greedy-Face) End-to-End routing process (EtE) reiterates until the final destination is reached. Simulation results show that EtE outperforms several existing geographical routing on energy consumption metric and delivery rate. Moreover, we prove that the computed path length and the total energy of the path are constant factors of the optimal for dense networks.
\end{abstract}

\section{Introduction}

Routing in WSN is a very challenging task due to the inherent characteristics that differentiates these networks from other networks like mobile ad hoc net- 
works or traditional IP-based networks. In sensor networks, a large number of sensors are generally deployed and because almost all applications of sensor networks require the flow of sensed data from at least one source to a particular base station or sink. Moreover sensor nodes are constrained in terms of energy, storage and processing capabilities therefore they require careful resource management.

In this paper, we focus on designing routing protocols that are scalable, energy efficient and that guarantee delivery in general network topologies. Centralized solutions generally need global knowledge, including position and activity status of all network nodes, thus nodes need the dissemination of route discovery information and need to maintain routing tables. To avoid this communication overhead, we focus on localized algorithms where only local information such as the position of the current node holding a packet, the one of its neighbors and the one of the destination are required.

Several localized routing protocols [1] with hop count as metric have been proposed to improve scalability. Each node has position information by using geographic positioning system (GPS) or other localization means [2] and routing decisions are made at each node using only local neighborhood information. Within this framework, energy-aware routing schemes use power consumption as metric and have been proposed in $[3,4,5,6,7,8]$.

Guaranteed delivery is another routing concern in sensor networks. Most routing schemes do not guarantee delivery especially in networks where obstacles such as holes and buildings are present. Several recovery schemes have been proposed [9] to overcome such a drawback.

Localized power aware routing algorithms that also guarantee delivery were proposed in $[5,7,8]$.

In this work, we propose an end-to-end geographic path discovery protocol $(E t E)$. To the best of our knowledge, it is the first position-based algorithm which at the same time guarantees packet delivery and reduces the energy consumption of both greedy and recovery parts. It satisfies the above objectives and more. It has the following characteristics:

- Localized: In EtE, to make a routing decision, a node has to be aware only of its location, of its neighbors and of the final destination.

- Scalable: EtE is memoryless as no routing information need to be stored at the node leading to a scalable protocol where no information is embedded in the message.

- Loop free: EtE is loop-free since the greedy step always chooses among its neighbors in the forwarding direction of the destination (nodes closer to the destination than itself). This makes any sender node $s$ on the path forward to a node closer to the destination than the sender node.

- Guarantees delivery: EtE has two routing phases. The first phase is a localized greedy protocol that is prone to routing failure. EtE guarantees delivery by invoking its second phase-Face routing when needed. 
- Energy efficient: Our simulations show that every routing step EtE takes is energy aware. To avoid expensive long edges, EtE computes an energy weighted localized shortest path (SP) from the relaying node to all its neighbors in the forward direction and selects the one that minimizes the cost of the SP to the progress towards the destination. To avoid expensive short edges, EtE runs Face routing over a connected dominating set (CDS) on which it computes a SP once again.

We prove that the total Euclidean length of the path found in greedy phase is within a constant factor of the optimum. For dense uniform networks, we also prove that the total energy of a computed path is constant factor of the optimal.

This paper is organized as follows. We introduce the network model and state our assumptions in Section 2. Then we briefly cover related work in Section 3. In Section 4, we introduce our protocol EtE. We compare EtE performance to existing protocols in Section 5 and conclude in Section 6.

\section{Preliminaries}

\section{$2.1 \quad$ Network Model}

While the network model can be arbitrary, the simulations are based on the widely adopted unit disk graph (UDG) model [10]. UDG is defined by $G=$ $(V, E)$, where $V$ represents the set of sensor nodes in the network and there is an edge $e=(u, v) \in E$ between nodes $u$ and $v$ if and only if the Euclidean distance between them $|u v| \leq R$, where $R$ is the transmission radius, equal for all nodes. Let $N(u)$ be the set of neighbors of node $u$. Let $\mathrm{N}_{x}(u)$ be the set of nodes from $N(u)$ which are closer to $x$ that is:

$$
\mathrm{N}_{x}(u)=\mathrm{N}(u) \cap\{\{v\} \text { s.t. }|x v|<|x u|\} .
$$

We define the density of the network as the average number of neighbors per node, also called the degree of a node noted $\delta: \delta(u)=\left|N_{x}(u)\right|$. We assume that each node is aware of its position and the one of its neighbors. This may be achieved through the use of a positioning device such as GPS ${ }^{1}$ or Galileo ${ }^{2}$. We assume that every node is aware of the position of the destination. This can be achieved thanks to the use of a location system such as in $[11,12]$.

\section{$2.2 \quad$ Energy Model}

The most common energy model is proposed in [13].

$$
\text { power }= \begin{cases}r^{\alpha}+c & \text { if } r \neq 0, \\ 0 & \text { otherwise. }\end{cases}
$$

\footnotetext{
${ }^{1}$ http://www.gps.gov/index.html

${ }^{2} \mathrm{http}$ ://ec.europa.eu/transport/galileo/index_en.htm
} 
where $r$ is the distance separating two neighboring nodes (a forwarding and a receiving node); $c$ is the overhead due to signal processing; $\alpha$ is a real constant $(\geq 2)$ that represents the signal attenuation.

This energy model implies that following only short edges may be as expensive as following long edges because of $c$. Note that, in reality, this needs to be multiplied with a constant that includes, for example, the message length. The optimal transmission radius, $r^{*}$, that minimizes the total power consumption for a routing task is equal to: $\mathrm{r}^{*}=\sqrt[\alpha]{\frac{\mathrm{c}}{\alpha-1}}$. We thus assume that nodes can adjust their range. The range adjustment may be performed by the link layer. ${ }^{3}$

\section{Literature review}

\subsection{Routing}

We briefly describe position based routing schemes relevant to this work. We distinguish between two routing metrics: hop count and power consumption.

\subsubsection{Hop count based routing}

In the greedy method [15], a node holding a packet forwards it to its neighbor $a$ that is closest to the destination. Though this greedy routing works well in dense networks, it fails if a node $a$ is closer to the destination than any of its neighbors. A routing algorithm that guarantees delivery in 2-D UDG is described in [1]. It applies greedy routing until either the message is delivered or the routing fails. In the latter case, Face routing is applied to recover from failure. Face routing requires the network topology to be a planar graph (i.e., no edges intersect each other). To planarize a graph, several algorithms can be used [16, 17]. Gabriel Graph (GG), for instance, contains edges between nodes $u$ and $v$ if and only if no other nodes are located inside the circle centered in the middle of edge $(u, v)$ and with diameter $|u v|$. GG has some desirable properties when used for routing in wireless networks such as localized message, free computation, planarity, and preserving connectivity [1]. GG divides the network into faces. The face that contains the line $(s d)$, where $s$ is the failure node, and $d$ is the destination node, is traversed by right-hand or left-hand rule (placing a virtual hand on the wall of the face) until a node $a$ closer to destination than $s$ is encountered. It has been shown in [9] that Face routing guarantees recovery traversing the first face. Greedy routing continues from $a$ until delivery or another failure node is encountered.

\footnotetext{
${ }^{3}$ The link layer may raise to the network layer the information which is necessary for the computation of the cost for each neighbor. Basically, the transmission power is sufficient to perform this cost. The evaluation of the required transmission power to reach a given neighbor is the difficult part of this task. Based on RSSI (received signal strength indicator) and LQI (link quality indicator) it is possible to perform this as shown in recent works[14].
} 


\subsubsection{Power consumption based routing}

Cost over Progress based routing $[18,3]$ is a localized metric aware greedy routing scheme. A node forwards a packet to the neighbor closer to $d$ such that the ratio of the energy consumed (any cost metric can be applied) to the progress made (measured as the reduction in distance to $d$ ) is minimized. Though energy efficient, this algorithm does not guarantee delivery.

The first power aware localized routing algorithms were described in [3]. Cost-over-progress framework with power as the cost has been applied in [19]. It was applied only in greedy phase and had similar performance to previous methods while enjoying simplicity in the design. The Iterative Power Progress algorithm, proposed in [19], is an improvement of the basic Power over Progress algorithm described in [19] as well. It works as follows. As in Power Progress, a node $s$,currently holding a message destined to $d$, first finds a neighbor $a$ that minimizes power $(r) /(|d s|-|d a|)$. Then, the search continues for an intermediate node $b$ which is: closer to $d$ than $s$, is neighbor to both $s$ and $a$, satisfies power $(|s b|)+\operatorname{power}(|b a|)<\operatorname{power}(\mid$ sa $\mid)$ and has the minimum power $(|s b|)+$ power $(|b a|)$ measure. If found, such node $b$ replaces $a$ as selected neighbor, and the search for a better intermediate node repeats. This process is iteratively repeated until no improvement is possible and node $s$ forwards the message to the selected neighbor, which then applies the same scheme for its own forwarding. The first article to address guaranteed delivery in power aware localized routing is [5]. It is a Greedy-Face-Greedy (GFG) approach where greedy routing is the same as in [3] while Face routing is similar to the one in [1]. One of the drawbacks of Face routing is that it is likely to follow short edges of GG that may be power inefficient.

GOAFR+[20] (Greedy Other Adaptive Face Routing Plus) is a combination of greedy routing and face routing. GOAFR+ presents two mechanisms of early fallback and boundary circle. Early fallback mechanism is to return from face routing mode to greedy forwarding mode as soon as possible. And the boundary circle is used to restrict a searching area in similar way as OAFR (Other Adaptive Face Routing) [6]. It reduces exploring a boundary of faces far away from the destination. These two mechanisms make GOAFR+ achieve optimal result in the worst-case. Then, some variants have been proposed to improve to find adapt circles boundary circles by using the location information of neighbors[21].

In [8], a GFG energy aware routing with guaranteed delivery has been proposed. The energy awareness is introduced at the greedy phase where the path to the selected neighbor is enhanced by following the energy weighted SP (with power consumption as weights). The algorithm works as follows. Node $s$ currently holding a packet selects neighbor $a$ closest to $d$ as its temporary destination node. Instead of transmitting directly to $a, s$ computes the energy weighted SP to $a$. This path is followed until a node $b$ closer to $d$ than $s$ is reached. To reach node $b$, the SP has to be embedded in the message, creating more overhead and making the protocol less energy efficient. Node $b$ then recursively applies the same protocol. Face routing [1] is applied to recover from failure points. In 
this work, we will refer to this protocol as SPFSP.

In LEARN [7], a localized energy aware routing is proposed. A node $s$ selects neighbor $b$ inside a restricted neighborhood $(\widehat{b s d} \leq \alpha$ for a parameter $\alpha<\pi / 3)$ that has the largest energy mileage, determined as the ratio $|s b| / \operatorname{power}(|s b|)$. If no such neighbor exists inside the restricted neighborhood, LEARN fails. In the variant LEARN-G, a node switches to greedy routing [15] in case of failure and selects the neighbor closest to the destination. Finally, in the variant LEARNGFG, a node invokes Face routing when a failure occurs.

Clearly, all existing algorithms use an energy unaware Face routing as a recovery routing scheme. To the best of our knowledge, this is the first work to combine Face routing with a power consumption metric.

\subsection{Connected dominating sets}

Dominating sets are defined as follows. Each node in a graph either belongs to a dominating set (DS) or has a neighbor in the DS. The problem of computing the smallest CDS is known to be NP-complete even if knowledge of the global topology is available. Dai and Wu [22] introduced a generalized DS concept, where coverage can be provided by an arbitrary number of connected one-hop neighbors. The definition was modified by [6], to avoid message exchanges between neighbors, to the following form. Node $a$ is covered by its one-hop neighbors $b, c, \ldots$ if these neighbors $b, c, \ldots$ are connected (any neighbor of $a$ is a neighbor of at least one node among $b, c, \ldots$ and $\operatorname{key}(a)<\min (k e y(b), k e y(c), \ldots))$. It is then further simplified in [23] as follows. First, each node checks if it is an intermediate node. Then each intermediate node $a$ constructs a subgraph $G^{\prime}$ of its neighbors with higher key values. If $G^{\prime}$ is empty or disconnected then $a$ is in the DS. If $G^{\prime}$ is connected but there exists a neighbor of $a$ which is not a neighbor of any node of $G^{\prime}$ then $a$ is in the CDS. If position information of 1-hop neighbors is available, nodes can decide whether or not they belong to a so defined CDS without exchanging any message with their neighbors. Note that these algorithms are local and do not induce any additional message overhead.

\subsection{Motivation}

In this paper, we aim at proposing a novel position-based algorithm which guarantees the packet delivery by reducing the energy consumption in both greedy and recovery steps.

\section{New Routing Approach - EtE Algorithm}

We describe a novel energy efficient georouting EtE with guaranteed delivery. It is based on a GFG routing in which both steps (greedy and Face) are energy aware. 


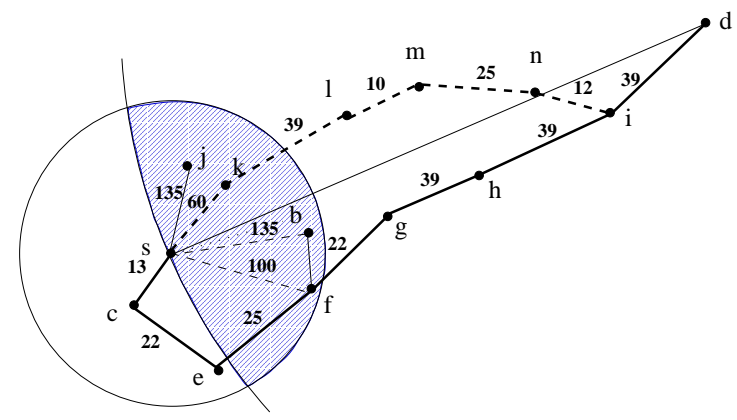

Figure 1: Greedy routing from node $s$ to $d$. In [8] $s$ selects $b$ and the truncated SP is $s-c-e-f$. EtE algorithm follows a path via node $k$.

\subsection{Greedy Routing}

The greedy step of EtE is based on the SP computation as in [8] but with important differences: first, in the choice of the temporary destination and second, in the computation of the SP. In [8], to send a message, a node $s$ first selects its neighbor node in its neighborhood which is the closest to the destination (node $b$ in Figure 1). Then it computes the SP between itself and the selected node. When node $s$ computes the energy weighted shortest path towards $b$, it considers all the nodes in its neighborhood (including nodes in backward direction $c, e$ and nodes in forward direction $b, f, j, k$ in Figure 1). In this example, the SP found goes through node $c$ which is not neighbor of the destination node $b$ and thus would not be able to compute the SP from itself to $b$. Therefore, the SP needs to be embedded in the message for the latter to reach node $b$ and avoid loops. In [8] the SP forwarding from $s$ to selected neighbor $b$ is truncated at node $f$, first node in the SP closer to $d$ than $s$.

In EtE, and contrarily to [8], the SP is computed only over nodes in the neighborhood of $s$ in forward direction (nodes closer to $d$ than to $s$ ). In Figure 1, $s$ considers only nodes in the grey shaded and striped area: nodes $b, f, j$ and $k$. Since each node on the SP that receives the message locally computes the next hop, EtE does not need to embed the SP in the message as in [8]. The message contains only the addresses of the source, destination and next hop selected neighbor of $s$. Since every node considers only nodes in the forwarding direction, there is no loop possible. As we show in our simulations, this is a major advantage of EtE over the method in [8] especially when the SP is long. Moreover, since at each step we get closer to the destination, it is clear that EtE is loop-free.

Let $\mathcal{F}(s, d)$ be a function that defines the selection criteria of $s$ 's neighbor $b$ on the path to $d$. Node $s$ selects node $b$ which minimizes $\mathcal{F}(s, d)$. Our greedy routing step differs from the one in [8] in the choice of $\mathcal{F}$. In [8], node $s$ selects its neighbor $b$ which is closest to the the destination $d$, i.e. $\mathcal{F}(s, d)=\min _{u \in \mathrm{N}_{d}(s)}|u d|$ which is satisfied for $|b d|=\mathcal{F}(s, d)$. 
To be more energy efficient, we propose to select this node in a cost-over progress fashion [19] where we define our cost as the SP cost. Let $x_{0}-x_{1}-\ldots-$ $x_{i}-x_{i+1}-. . x_{n}$, be the nodes on the SP from $s$ to $b$ with $x_{0}=s$ and $x_{n}=b$. We define the cost $\operatorname{cost}_{S P}(s, b)$ of the SP from $s$ to $b$ as

$$
\operatorname{cost}_{S P}(s, b)=\sum_{i=0}^{n-1} \operatorname{power}\left(\left|x_{i} x_{i+1}\right|\right) .
$$

Node $s$ then selects node $b$ which minimizes the cost of the SP from $s$ to $b$ divided by the progress it makes towards destination node $d . \mathcal{F}(s, d)$ is then expressed as $\mathcal{F}(s, d)=\min _{u \in \mathrm{N}_{d}(s)} \frac{\operatorname{cost}_{S P}(s, u)}{\| s d|-| u d||}$. In Figure 1, the neighbor that would be selected by the algorithm proposed in [8] is $b$ while EtE would select node $k$. Note that in the given example, the SP from $a$ to $k$ is that link itself, which may happen frequently because of cost-over-progress optimality criteria for selecting neighbors. In other examples, intermediate nodes may be used on the SP to reduce the overall energy. In such a case, node $s$ will send the message to the first node $a$ on the SP towards the selected node $b$, and $a$ will apply the same procedure which is formally described in Algorithm 1.

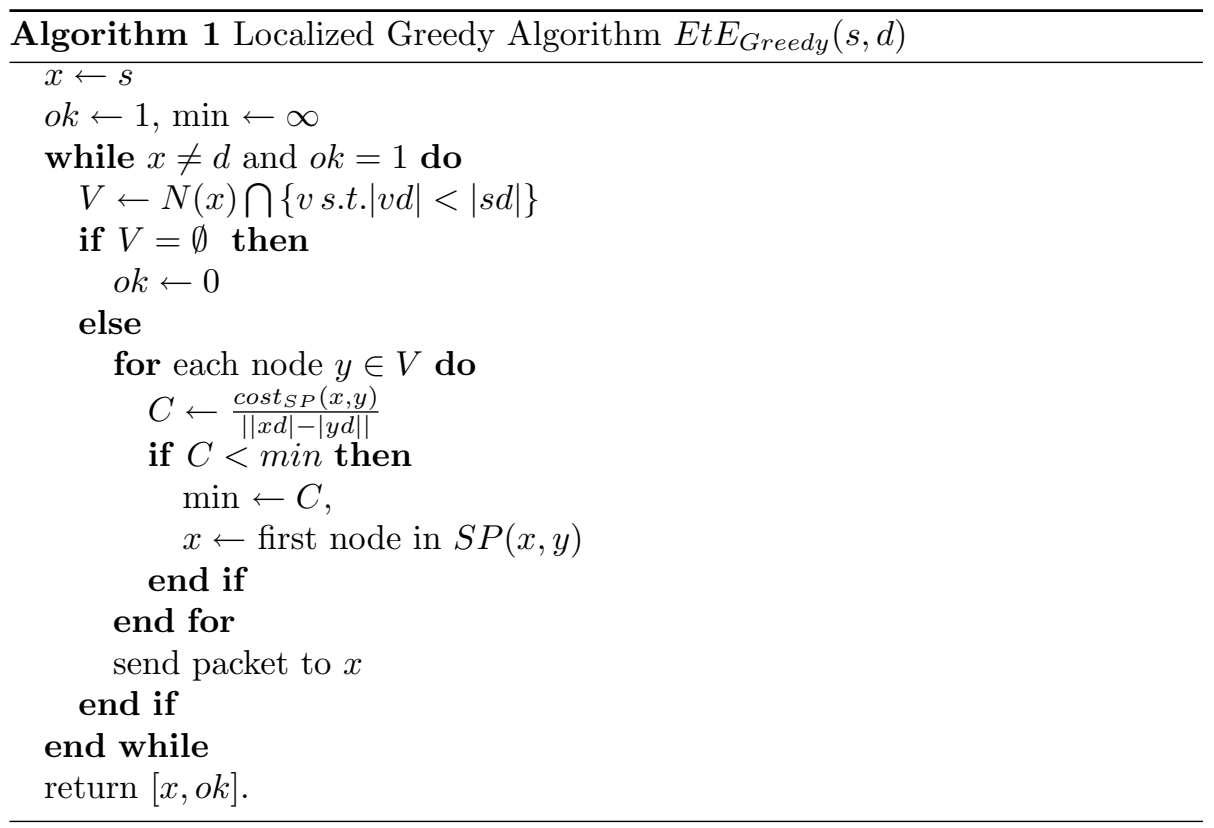

\subsection{Recovery Step Routing}

Regular Face routing guarantees delivery, but is not energy efficient since it may use very long as well as very short edges compared to the energy optimal transmission length $r^{*}$. To overcome this drawback, we introduce in this work 
an energy efficient variant to Face routing. From the original graph $G=(V, E)$ (Figure 2(a)) we compute a CDS, $V^{\prime}$ of $V$. Since source $s$ and destination $d$ may not be in the CDS, we expand $V^{\prime}$ by adding them to this set. Let $G^{\prime}=\left(V^{\prime}, E^{\prime}\right) \subset G$ where $V^{\prime} \subset V$ is the expanded set of dominant nodes and $E^{\prime} \subset E$ is the set of edges between nodes in $V^{\prime}$. We use the CDS election protocol introduced in [23], however other election protocol may be applied. Since Face routing must be applied on a planarized graph, we generate the GG, $G^{\prime \prime}=\left(V^{\prime}, E^{\prime \prime}\right)$, from $G^{\prime}$ where $E^{\prime \prime} \subset E^{\prime}$ is the set of edges remaining in the planarized graph (Figure 2(c)). Note that the computation of a GG is totally local. We then run Face routing over $G^{\prime \prime}$. Face routing guarantees delivery in the constructed subset since it contains source and destination nodes and preserves connectivity. Moreover, by considering only edges connecting two dominating nodes, the routing process avoids to choose too short edges. Each node needs to know its neighbors that are in the CDS. This may come from 2-hop position knowledge, or by adding a bit in any message sent by nodes to their neighbors. However, the former approach will increase the communication overhead significantly, and such strong assumptions are not used in competing algorithms. The later approach will have minor impact on the overall overhead, and can function with 1-hop positional knowledge. Alternatively, one can rely on beaconless routing that guarantees delivery [24]. We will not elaborate on the details of the protocol. In brief, current node $s$ that is in recovery mode will send a request to its neighbors to continue face traversals. Only neighbors that belong to the constructed CDS may respond. In the simple solution, all neighbors respond to allow $s$ to select the proper edge in GG of dominant nodes. Such messages use short packets that consume negligible energy compared to messages needed to transmit a full message that is being relayed, therefore they are ignored in our analysis. Solutions with reduced number of responses are described in $[24,25]$.

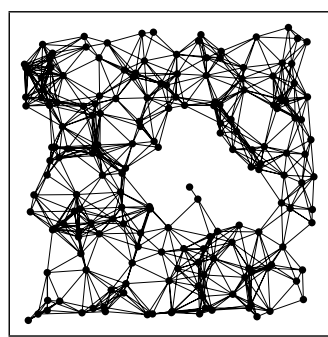

(a) Initial graph $G$

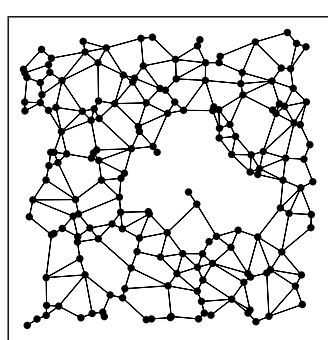

(b) GG on $G$

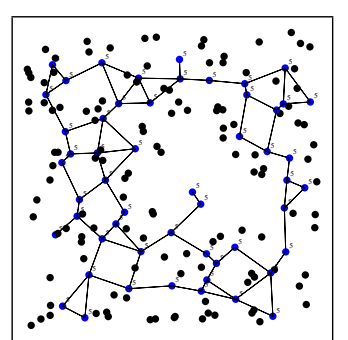

(c) $G^{\prime \prime}=$ GG from $G^{\prime}$

Figure 2: When using regular Face routing over Graph $G(a)$, messages follow GG edges of $G(b)$. Edges of $G$ " $(c)$ are used instead when applying our energyefficient Face routing.

Application of Face routing on $G^{\prime \prime}$ only decides on which edge $(s, b)$ to follow to reach the destination node from a given node $s$. However, that edge does not need to be selected since it may be too long $\left(|s b|>r^{*}\right)$. Therefore, we 
apply once again an energy weighted SP to reach node $b$. It is the greedy algorithm described in Algorithm 1, where final destination node $d$ is replaced by temporary destination $b$. That is, $E t E_{\text {Greedy }}(s, b)$ is invoked. Once node $b$ is reached, we follow the EtE routing heuristic. If $b$ is closer to the destination node than the node which has initiated Face routing step, node $b$ selects the next hop in the routing path following the greedy routing described above. Otherwise, it determines the node following Face routing over CDS nodes and computes the energy based shortest path to reach it. This process reiterates until the destination is reached. This procedure is formally described in Algorithm 2.

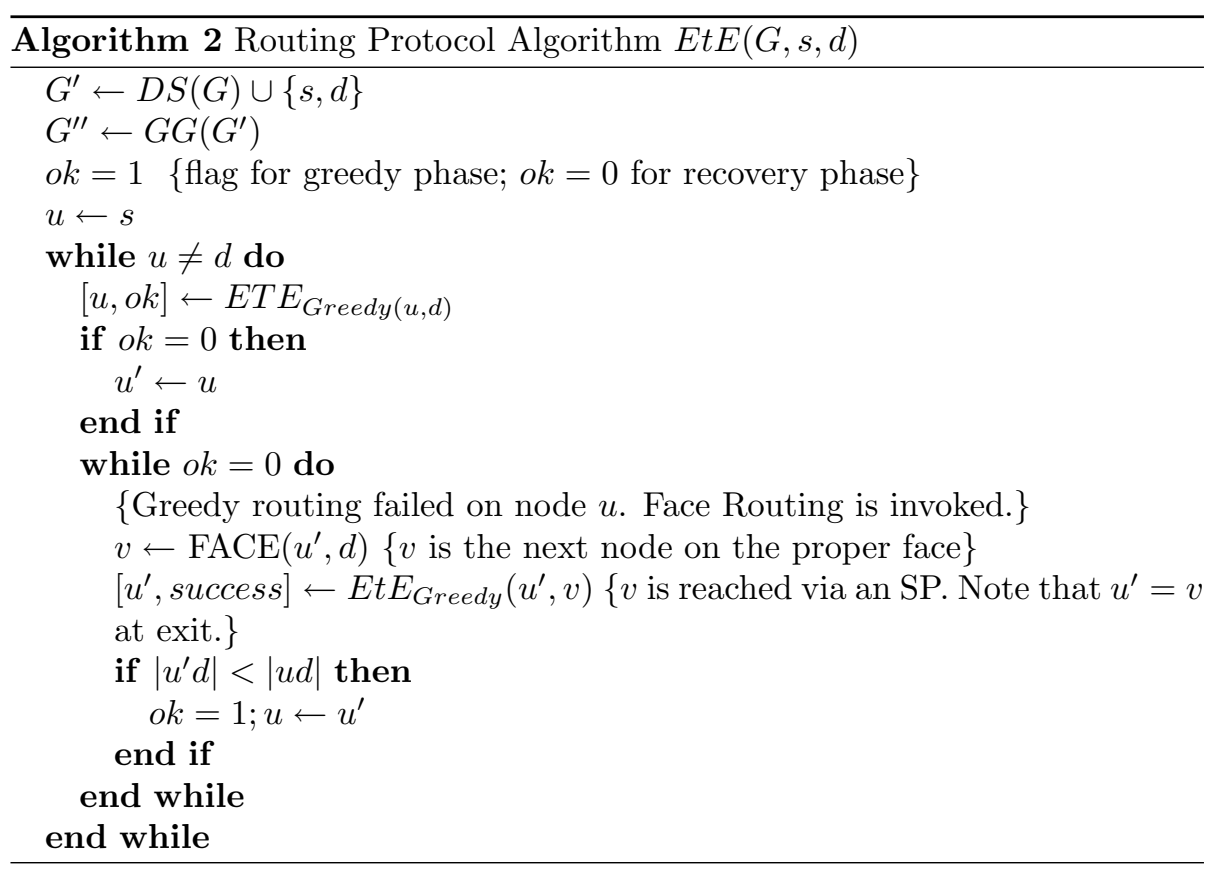

Figure 3 illustrates a sample execution of EtE algorithm. Greedy routing proceeds from node 1 which first computes the cost of SP towards nodes 2, 19 and 21 (node 23 is not included in the selection since it is further from the destination than node 1 ). Node 1 selects node 19 as temporary destination since it provides the lowest cost over progress and sends the packet to node 21, the first node on the SP towards 19. Node 21 finds node 20 as its best forwarder and in this case SP is that link. Node 20 selects node 18 by following a SP through node 16 to which it sends the message. The latter then forwards to node 18 where greedy routing fails. Face routing is then invoked to follow edges 18-16 (directly), 16-15 and 15-11 (which are replaced by paths 16-14-15 and 15-13-11 respectively for energy efficiency). Greedy routing then continues till delivery to 11 selecting 10 via 9,9 selecting 7 via 10, and 10 selecting destination 8 and delivering via node 7 .

We now prove more properties of EtE algorithm. 


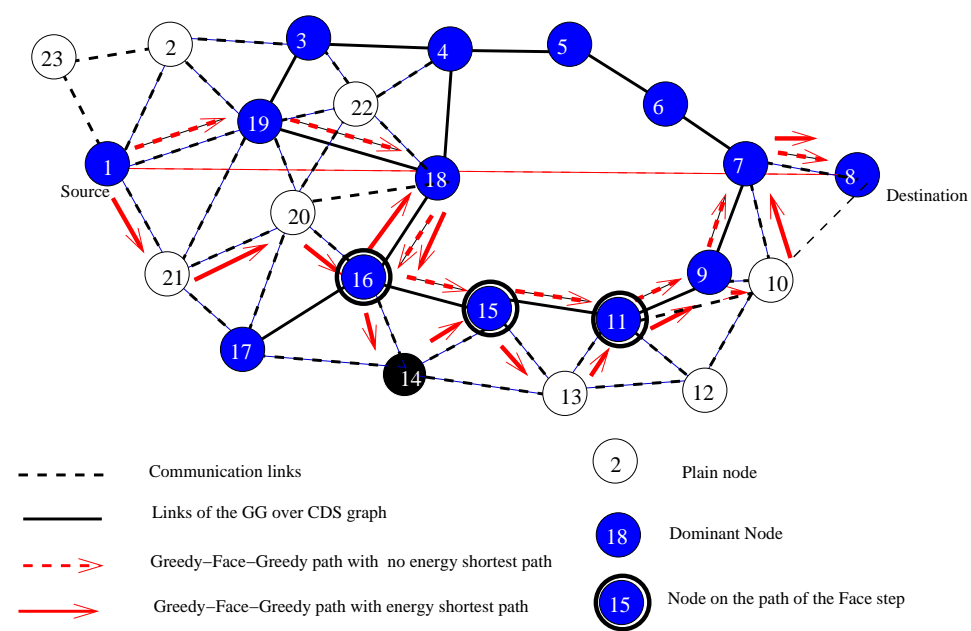

Figure 3: Illustrative example of EtE algorithm on a sample network.

Definition 1 A path meets angular constraints if every hop is within an angle $\theta \leq \alpha<\frac{\pi}{3}$ toward the destination node.

Theorem 1 Any path from source node $s$ to destination node $d$ that meets angular constraint has length that is constant length of the optimum $|s d|$.

Proof 1 The proof is the same as in [7] where it was restricted specifically to the protocol LEARN. Since that proof does not include any cost function, and is based solely on geometric arguments, it is generally valid for a family of protocols respecting the angular constraint.

Theorem 2 When EtE routing finds a path from source $s$ to destination $d$ in dense graphs, the total energy consumption of the path is within a constant factor of the optimum.

Proof 2 For any intermediate node $s$ with packet to forward, EtE routing protocol selects the neighbor $u$ such that $\frac{\text { power }_{s, u}}{|s d|-|d u|}$ is minimized. In [Y], the neighbor $v$ is selected such that $\frac{\text { power }_{s, v}}{|s v|}$ is minimized.Comparing the cost to progress provided by both choices it then follows that $\frac{\text { power }_{s, u}}{|s d|-|d u|} \leq \frac{\text { power }_{s, v}}{|s d|-|d v|}$. The expected number of hops by both algorithms are $\frac{|s d|}{|s d|-|d u|}$ and $\frac{|s d|}{|s d|-|d v|}$ respectively. The expected energy consumption of the respective paths are then equal to $\left\{\right.$ power $\left._{s, u} \cdot \frac{|s d|}{|s d|-|d u|}\right\}$ and $\left\{\right.$ power $\left._{s, v} \cdot \frac{|s d|}{|s d|-|d v|}\right\}$ which is equivalent to multiplying both sides of the above inequality by $\mid$ sd $\mid$. Thus we get $\left\{\right.$ power $\left._{s, u} \cdot \frac{|s d|}{|s d|-|d u|}\right\} \leq$ $\left\{\right.$ power $\left._{s, v} \cdot \frac{|s d|}{|s d|-|d v|}\right\}$. Therefore EtE consumes less energy than LEARN. Since LEARN was proven in [7] to require constant factor of the optimum power for sufficiently dense networks, the theorem follows. Note that the argument is in fact probabilistic with details given in [7]. 


\section{Performance Evaluation}

We compare several protocols via simulation. We use WSNet/Worldsens [26] event-driven simulator for large scale wireless sensor networks, that assumes 802.11 DCF MAC layer, free space propagation model and packet collisions. For the purpose of the simulation we have created 20 different networks which can be described as follows. Nodes are randomly deployed in a $1000 \times 1000$ square using a Poisson Point Process (node positions are independent) with different mean node degree $\delta^{4}$. These nodes have the same maximum transmission range, $R=200$ but can adapt their transmission range between 0 and $R$. We compare the performance of EtE to existing geographic routings GFG [9], SPFSP [8] and LEARN-G [7]. We run the simulator for the same samples of node distribution, same source node and destination node, both randomly chosen. For every of 20 different network topologies we run around 150 depending on network size. We evaluate the energy consumption of each algorithm based on the energy model described in Section 2. As in [13], we use $c=3 \cdot 10^{7}$ and $\alpha=4$, which leads to an optimal routing edge length of 100 [19]. The results obtained are within a 95\% - confidence interval. To further evaluate the routing protocols, we computed their energy overhead using as reference the optimal centralized energy weighted $\mathrm{SP}$ (Dijkstra algorithm [27]). We let $e_{i}$ and $e^{*}$ be the energy consumed to route a packet from $s$ to $d$ using any one of the described protocol that guarantees delivery and the centralized SP protocol, respectively. We define the energy overhead as the ratio $\frac{e_{i}-e^{*}}{e^{*}}$. Since one of the novelty of EtE is the use of a CDS, we compare it to its variant EtE' that uses the basic Face phase as in SPFSP. We evaluate the performance of each phase of the protocols independently for better insights on the behavior of the routing schemes. Finally, to insure that Face routing is invoked, we evaluate the performance of the routing schemes on a homogeneous network (no holes and no obstacles) and on a topology with a crescent hole (see Fig. 2(a)).

\subsection{Routing paths power consumption}

Figs. 4 and 5 show that our algorithms EtE and EtE' outperform existing solutions. They consume only $4 \%$ more energy than the optimal centralized algorithm in a homogeneous environment (Fig. 4) and only $12 \%$ more energy in a topology with a hole (Fig. 4). The next best performing algorithm is LEARN$\mathrm{G}$ followed by SPFSP. The worse results are achieved by GFG which consumes $55 \%$ more energy than the optimal solution. Since EtE and EtE' perform the same greedy algorithm, EtE outperforms EtE' only when Face routing is usedin networks with low density and/or with obstacles. In dense networks greedy routing almost always succeeds (see Section 5.2).

\footnotetext{
${ }^{4}$ In such a Poisson Point Process, the total number of nodes is probabilistic and is obtained from a Poisson Law of intensity $\lambda$ with $\lambda=\frac{\delta}{\pi R^{2}}$.
} 


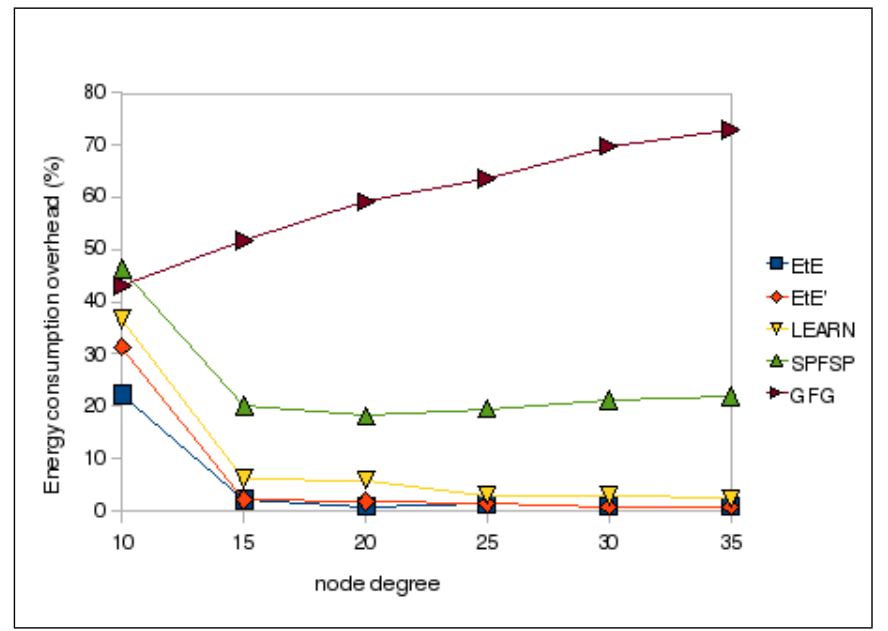

Figure 4: Energy consumption in a homogeneous network.

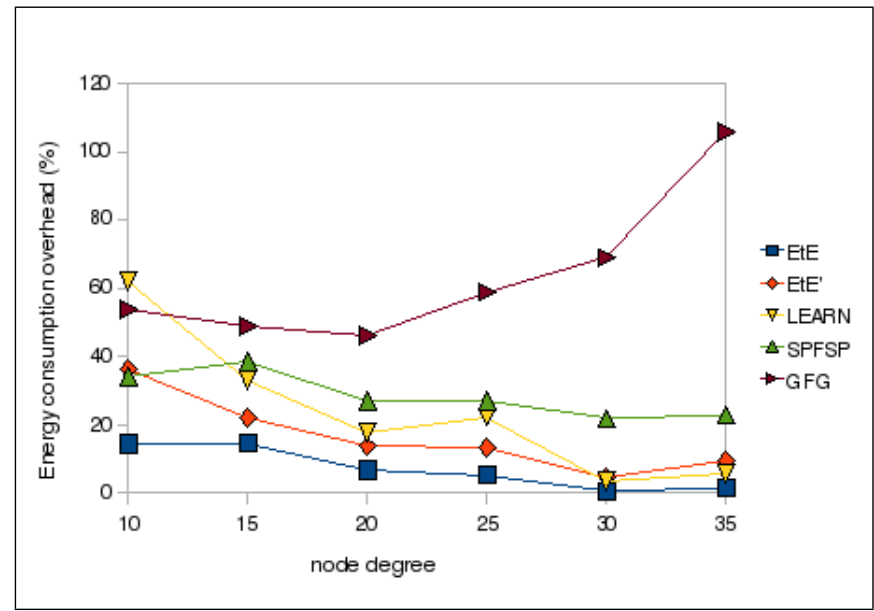

Figure 5: Energy consumption in a topology with obstacles. 


\subsection{Greedy routing performance evaluation}

Our simulations show that greedy routing achieves almost $100 \%$ success rate in a homogeneous network when the density is higher than 15. For the topologies with a hole the success rate is slightly below $100 \%$ for mean node degree lower than 30. Figs. 6 and 7 plot the energy overhead for both topologies (with and without a hole). The overhead is computed only for successful routings. Our simulations show that our proposed greedy routing algorithm outperforms existing solutions. Moreover, privileging nodes within an angular sector toward the destination and favoring the one with the better energy to distance ratio as in LEARN is more efficient than computing a SP toward the node that minimizes the remaining distance to the destination. GFG is the solution which offers the worst performing greedy algorithm, followed by SPFSP and LEARN.

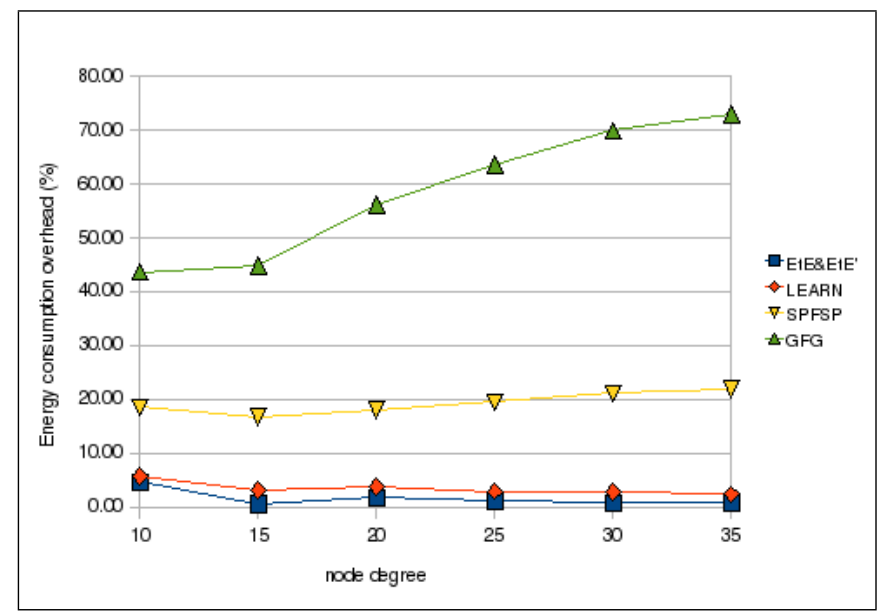

Figure 6: Greedy energy consumption in a homogeneous network.

\subsection{Face routing performance evaluation}

We evaluate four variants of Face routing algorithm independently of the greedy phase: (1) Face used by GFG, run on a GG issued from the entire set of nodes in the network; (2) SPFACE, used by EtE' and SPFSP which selects the next node using Face and reaches it by performing an energy weighted SP; (3) DSFACE, used by LEARN-G, run over a GG issued from the CDS; and (4) SPDSFACE, used by EtE, which selects the next node using DSFACE and reaches it by performing an energy weighted SP. Our results are given in Fig. 8. It is interesting to notice that by using a CDS, we achieve better energy saving. This can be explained by the fact that when extracting a GG from the entire set of nodes in the network, we keep only short edges, often smaller than the optimal hop length. So, there is no need to perform an energy weighted SP. When extracting a GG from a CDS, we keep edges which may easily be longer or equal to 


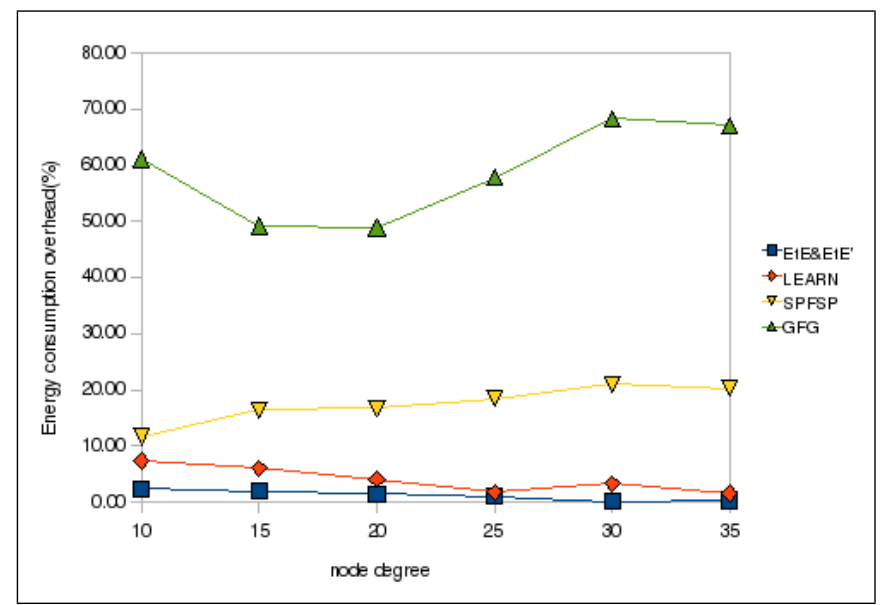

Figure 7: Greedy energy consumption in a network with obstacles.

the optimal hop length. In this case, performing an energy weighted SP is of interest. Fig. 10 plots face edges average length for the different GGs and the length of the edges followed by the various Face routing algorithms. We notice that SPFACE and Face obtain exactly the same results.

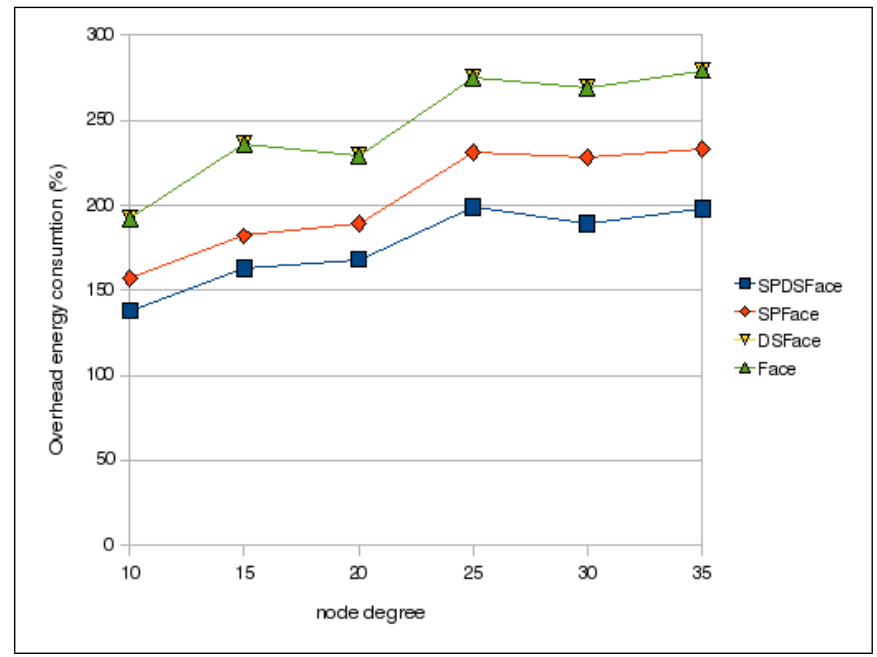

Figure 8: Face routing features of the different routing protocols.

It is interesting to note that introducing a CDS before planarizing the graph allows the computation of more energy efficient routes for any node density and range values. However, edge length of the planarized graph depends on the maximum radius $R$ (see Fig. 9). Clearly, for a node density of 10, $R<150$ and using a CDS, Fig. 9 shows that face edges are smaller than $R_{o p t}$. Therefore, 
there is no need to use the SP on Face routing.

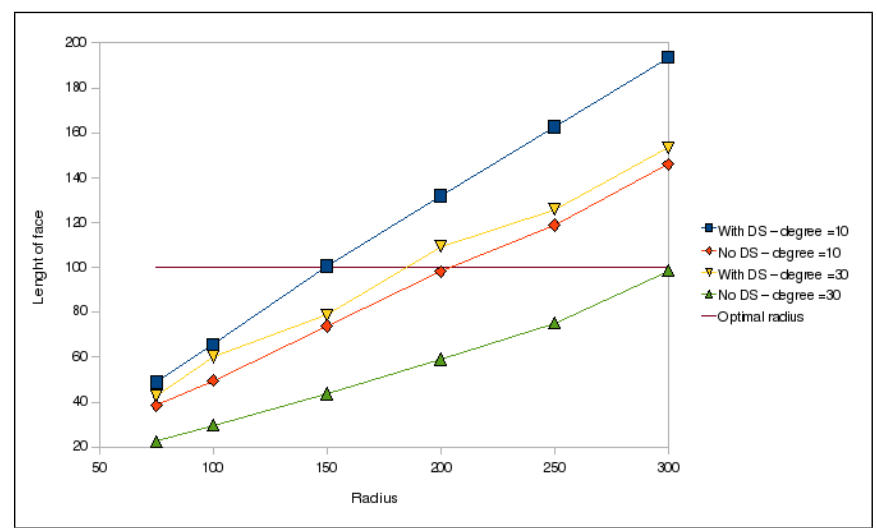

Figure 9: Relationship between face edges length and $R$ for the GGs, when $\delta=10$ and 30 .

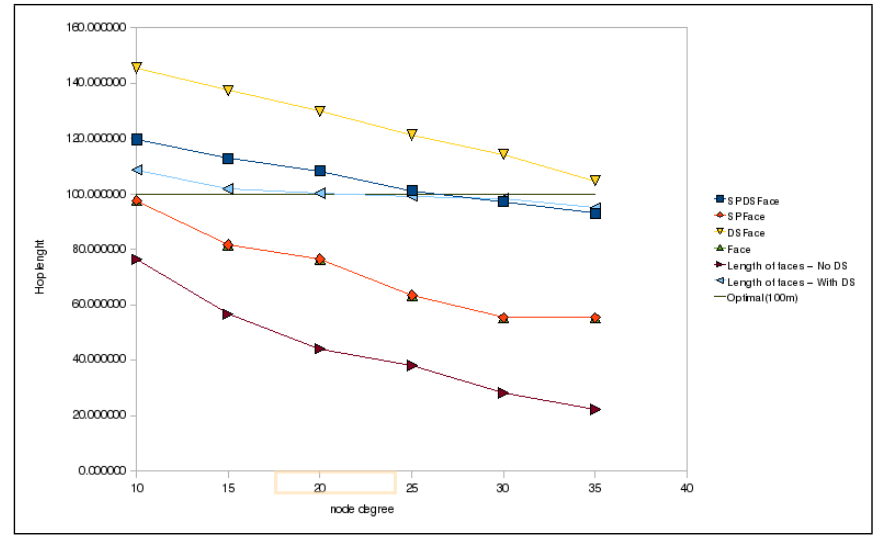

Figure 10: Edges length for face in both GGs and for the routing paths.

Fig. 10 shows that face edges length in the GG extracted from the DS are independent of $\delta$. However, this is not true for the GG issued from the entire network. The figure supports our previous claim. It plots, for a given $\delta$, the range of $R$ values for which it is worth using a CDS. Moreover, Fig. 11 shows that energy savings are achieved for values of face edges length lower than $R_{\text {opt }}$. The plot is for $\delta=30$, but the same results are obtained for various values of $\delta$.

\subsection{Enlarging the network}

So far, we evaluated and compared several routing algorithms on a fixed network size while varying the network density. In this section, we focus on studying the effect of the network size on the performance of the various protocols. We fix 


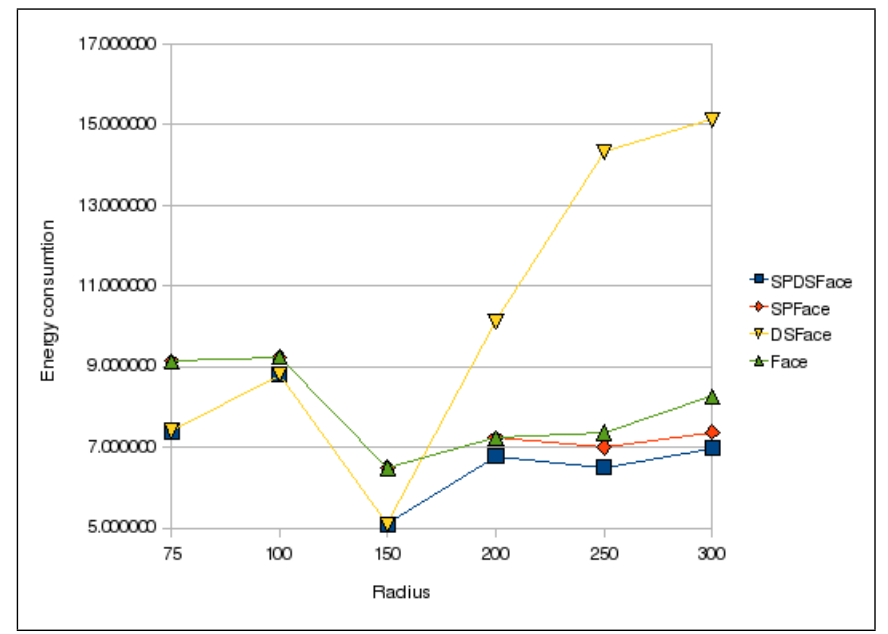

Figure 11: Energy consumption as a function of $R$.

the node density to $\delta=15$ and the maximum node range radius to $R_{\max }=200$. Figs. 12 and 13 plot the energy consumption overhead of the routes computed by each algorithm as a function of the network size.

Clearly, as the network size increases, so does the computed path length. For homogeneous networks, we notice that the energy overhead consumed by each protocol is pretty constant which makes it independent of the network size. This can be explained by the fact that for dense networks, greedy routing almost always succeeds and Face routing is rarely invoked. On the other hand, we notice that for network topology with obstacles, the energy overhead tends to slightly increase as the network size increases. This is due to the fact that the larger the network (and the longer the paths), the higher the chances for greedy routing to encounter a dead end and invoke Face routing. Moreover, Face routing has an energy overhead higher than greedy routing energy overhead (see Sections 5.2 and 5.3).

We also compared GOAFR + performance to EtE via simulation. Our results confirm that EtE outperforms GOAFR+. The latter applies standard greedy phase and a variation of FACE recovery phase and makes use of several parameters which impact its performances. For instance, the parameter $\sigma$ affects directly the behavior of the recovery phase. In fact for small values of $\sigma-e . g$. $1 / 3$ as recommended by the authors-, the recovery phase is very similar to the original FACE. GOAFR+ is efficient when the considered network has big holes. However, our simulation results, with a value of $\sigma=1 / 100$ (like used by the authors in their simulations), show that EtE is $28 \%$ more energy efficient than GOAFR + for sparse networks (10 nodes per communication area). For dense networks, the energy consumption of GOAFR+ is similar to GFG presented in previously. 


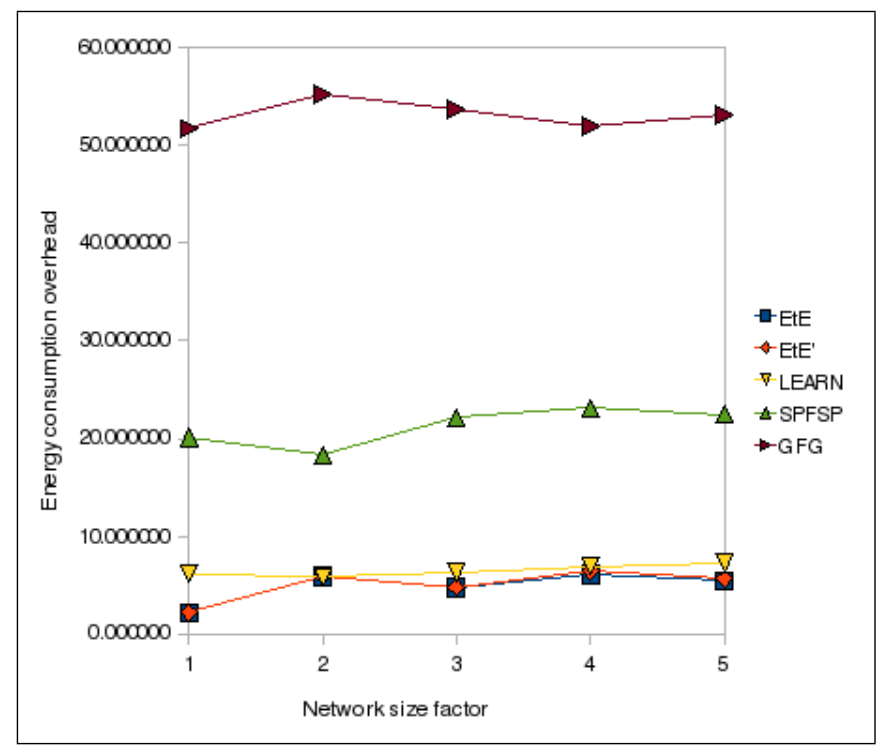

Figure 12: Energy consumption overhead in a homogeneous network when $R_{\max }=200$ and $\delta=15$. The $\mathrm{x}$-axis represents the network size multiplicative factor.

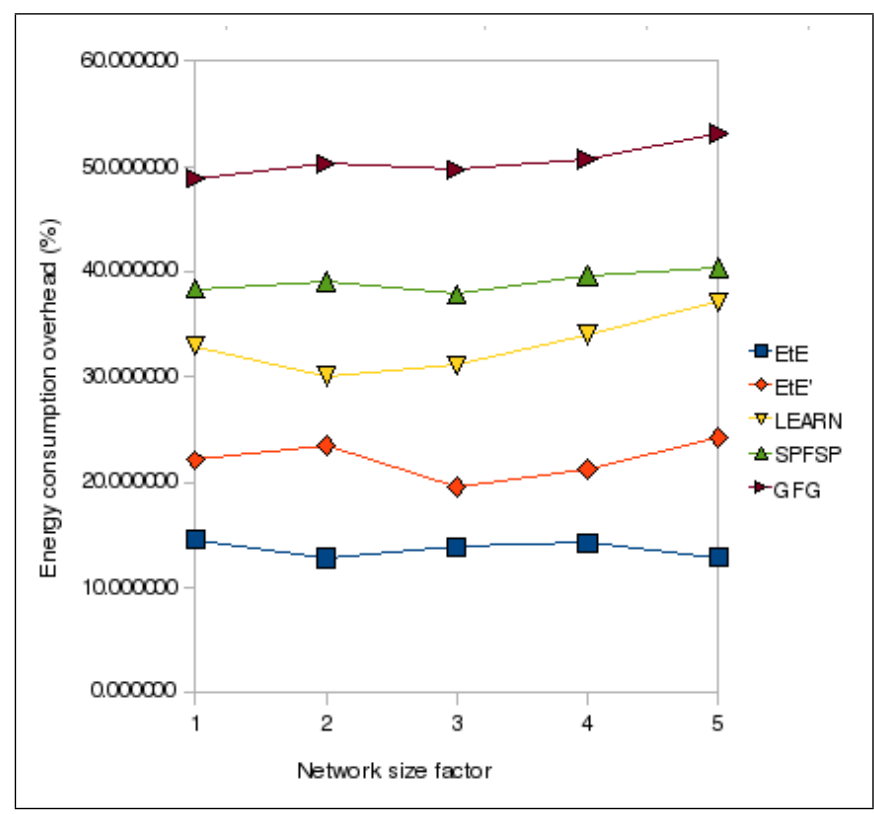

Figure 13: Energy consumption in a network with obstacles when $R_{\max }=200$ and $\delta=15$. The $\mathrm{x}$-axis represents the network size multiplicative factor. 


\section{Conclusion}

We propose a novel routing protocol, EtE, that is both energy efficient and guarantees delivery. EtE is general enough to be efficiently deployed in a network with arbitrary topology (networks with obstacles and holes). It is a two-phase routing protocol that uses an energy optimized greedy routing followed by an energy efficient Face routing scheme when a local minima is encountered. To the best of our knowledge, EtE is the first and only algorithm that uses an energy efficient Face routing. It is achieved by the integration of the dominating set to optimize the paths edge length used in the routing process. Based on our intensive simulations, EtE outperforms various energy-aware routing schemes. EtE considers a well-used energy cost model but it is worth noting that this cost computation can be replaced by any metric cost that one wishes to optimize. For instance, the cost of a link may be the delay introduced by using that link. Yet, EtE is also a general algorithm that can be used to enhance any metric.

Next steps of this work will be the analysis of the energy cost for maintaining the Face graph.

\section{$7 \quad$ Acknowledgments}

This research was partially supported by NSERC Canada, a grant from CPER Nord-Pas-de-Calais/FEDER TAC COMDOM, from an INRIA ARC program CARMA and from the French National Research Agency RNRT SVP (Supervise and Protect $)^{5}$.

\section{References}

[1] P.Bose, P. Morin, I. Stojmenovic, and J. Urrutia, "Routing with guaranteed delivery in ad hoc wireless networks," in Proc. 3rd Int. Workshop on Discrete Algorithms and Methods for Mobile Computing and Comm. (DIAL-M), Seattle, WA, USA, August 1999, pp. 48-55.

[2] E. H. Elhafsi, N. Mitton, and D. Simplot-Ryl, "Cost over progress based energy efficient routing over virtual coordinates in wireless sensor networks," in Proc. IEEE International Workshop: From Theory to Practice in Wireless Sensor Networks (t2pWSN 2007), Helsinki, Finland, 2007.

[3] I. Stojmenovic and X. Lin, "Power-aware localized routing in wireless networks," IEEE Trans. Parallel Distrib. Syst. (TPDS), vol. 12, no. 11, pp. 1122-1133, 2001.

[4] E. H. Elhafsi and D. Simplot-Ryl, "Flattening the gap between sourcedestination paths in energy efficient greedy georouting in wireless sensor

\footnotetext{
${ }^{5}$ http://svp.irisa.fr/
} 
networks," in 3nd Int. Conference on Mobile Ad-hoc and Sensor Networks MSN'07, December 2007.

[5] I. Stojmenovic and S. Datta, "Power and cost aware localized routing with guaranteed delivery in wireless networks," Wireless Comm. and Mobile Compt., vol. 4, no. 2, pp. 175-188, 2004.

[6] D. Simplot-Ryl, I. Stojmenovic, and J. Wu, "Energy efficient backbone construction, broadcasting, and area coverage in sensor networks," Handbook of Sensor Networks: Algorithms and Architectures, Wiley, pp. 343-379, 2005.

[7] Y. Wang, W.-Z. Song, W. Wang, X.-Y. Li, and D. T.A., "Learn: Localized energy aware restricted neighborhood routing for ad hoc networks," Sensor and Ad Hoc Communications and Networks, 2006. SECON06. 2006 3rd Annual IEEE Communications Society, vol. 2, pp. 508-517.

[8] J. A. Sanchez and P. M. Ruiz, "Exploiting local knowledge to enhance energy-efficient geographic routing," in 2nd Int. Conference on Mobile Adhoc and Sensor Networks MSN'06, December 2006, pp. 567-578.

[9] H. Frey and I. Stojmenovic, "On delivery guarantees of face and combined greedy-face routing in ad hoc and sensor networks," in Proc. of the Twelfth ACM Annual Int. Conference on Mobile Computing and Networking (MOBICOM), Los Angeles, Los Angeles, CA, USA, September 2006, pp. 390-401.

[10] B. Clark, C. Colbourn, and D. Johnson, "Unit disk graphs," vol. 86, no. 1-3, pp. 165-177, 1990.

[11] J. Li, J. Jannotti, D. D. Couto, D. Karger, and R. Morris, "A scalable location service for geographic ad hoc routing," in Proc. of the Sixth ACM Annual Int. Conference on Mobile Computing and Networking (MOBICOM), Los Angeles, Boston, MA, USA, August 2000.

[12] I. Stojmenovic, "Location updates for efficient routing in ad hoc networks," Handbook of wireless networks and mobile computing, pp. 451-471, 2002.

[13] V. Rodoplu and T. Meng, "Minimizing energy mobile wireless networks." IEEE Journal on Selected Areas, vol. 17, no. 8, pp. 1333-1347, 1999.

[14] F. Khadar and D. Simplot-Ryl, "From theory to practice: topology control in wireless sensor networks." in Proc. of the 10th ACM International Symposium on Mobile Ad Hoc Networking and Computing (MobiHoc), New Orleans, USA, 2009, poster abstract.

[15] G. Finn, "Routing and addressing problems in large metropolitan-scale," Internetworks, ISI Research Report ISU/RR-87-180, March 1987. 
[16] J. Li, L. Gewali, H. Selvaraj, and V. Muthukumar, "Hybrid greedy/Face routing for ad-hoc sensor network," $d s d$, vol. 0, pp. 574-578, 2004.

[17] G. Toussaint, "The relative neighborhood graph of a finite planar set," Pattern Recognition, vol. 12, no. 4, pp. 261-268, 1980.

[18] I. Stojmenovic, "Localized network layer protocols in sensor networks based on optimizing cost over progress ratio," IEEE Network, vol. 20, pp. 21-27, January/February 2006.

[19] J. Kuruvila, A. Nayak, and I. Stojmenovic, "Progress and location based localized power aware routing for ah hoc sensor wireless networks," IJDSN, vol. 2, pp. 147-159, 2006.

[20] "Geometric ad-hoc routing: Of theory and practice," in Proc. of The 22nd ACM International Symposium on The Principles of Distributed Computing (PODC), 2003, pp. 63-72.

[21] "GOAFR plus-ABC: Geographic routing based on adaptive boundary circle in manets," in International Conference on Information Networking (ICOIN), 2009, pp. 1-3.

[22] F. Dai and J. Wu, "An extended localized algorithm for connected dominating set formation in ad hoc wireless networks," IEEE Trans. Parallel and Distributed Systems (TPDS), 2004.

[23] J. Carle and D. Simplot-Ryl, "Energy efficient area monitoring by sensor networks," IEEE Computer Magazine, vol. 37, pp. 40-46, 2004.

[24] H. Kalosha, A. Nayak, S. Ruehrup, and I. Stojmenovic, "Select and protest based beaconless georouting with guaranteed delivery in wireless sensor networks," IEEE INFOCOM, Phoenix, Arizona, USA, April 13-18 2008 (to appear).

[25] M. Chawla, N. Goel, K. Kalaichelvan, A. Nayak, and I. Stojmenovic, "Beaconless position-based routing with guaranteed delivery for wireless ad hoc and sensor networks," Acta Automatica Sinica, vol. 32, no. 6, November 2006 .

[26] A. Fraboulet, G. Chelius, and E. Fleury, "Worldsens: Development and prototyping tools for application specific wireless sensors networks," in IPSN'07 Track on Sensor Platforms, Tools and Design Methods (SPOTS). Cambridge, Massachusetts, USA.: ACM, April 2007.

[27] E. Dijkstra, "Solution of a problem in concurrent programming control," Communications of the ACM, vol. 8, no. 9, September 1965. 